\title{
Changes in fungal community composition of biofilms on limestone across a chronosequence in Campeche, Mexico
}

\section{Cambios en la composición de la comunidad fúngica de biopelículas sobre roca calcárea a través de una cronosecuencia en Campeche, México}

\author{
Sergio Gómez-Cornelio’,, Otto Ortega-Morales², Alejandro Morón-Ríos', Manuela Reyes-Estebanez² and Susana de la \\ Rosa-García ${ }^{3}$
}

1 El Colegio de la Frontera Sur, Av. Rancho polígono 2A, Parque Industrial Lerma, 24500 Campeche, Mexico.

2 Universidad Autónoma de Campeche, Departamento de Microbiología Ambiental y Biotecnología, Avenida Agustín Melgar s/n, 24039 Campeche, Mexico.

3 Universidad Juárez Autónoma de Tabasco, División Académica de Ciencias Biológicas, Carretera Villahermosa-Cárdenas km 0.5 s/n, entronque a Bosques de Saloya, 86150 Villahermosa, Tabasco, Mexico.

4 Author for correspondence: sgomez@ecosur.edu.mx

To cite as

Gómez-Cornelio, S., O. Ortega-Morales, A. Morón-Ríos, M. Reyes-Estebanez y S. de la Rosa-García. 2016. Changes in fungal community composition of biofilms on limestone across a chronosequence in Campeche, Mexico. Acta Botanica Mexicana 117: 59-77.

Received: 28 de marzo de 2016. Reviewed: 6 de julio de 2016. Accepted: 2 de septiembre de 2016

\section{ABSTRACT:}

Background and Aims: The colonization of lithic substrates by fungal communities is determined by the properties of the substrate (bioreceptivity) and climatic and microclimatic conditions. However, the effect of the exposure time of the limestone surface to the environment on fungal communities has not been extensively investigated. In this study, we analyze the composition and structure of fungal communities occurring in biofilms on limestone walls of modern edifications constructed at different times in a subtropical environment in Campeche, Mexico.

Methods: A chronosequence of walls built one, five and 10 years ago was considered. On each wall, three surface areas of $3 \times 3 \mathrm{~cm}$ of the corresponding biofilm were scraped for subsequent analysis. Fungi were isolated by washing and particle filtration technique and were then inoculated in two contrasting culture media (oligotrophic and copiotrophic). The fungi were identified according to macro and microscopic characteristics.

Key results: We found 73 genera and 202 species from 844 isolates. Our results showed that fungal communities differed in each biofilm. In the middle-aged biofilm a high number of isolates was found, but both species richness and diversity were low. In contrast, in the old biofilm species richness and diversity were high; Hyphomycete 1, Myrothecium roridum and Pestalotiopsis maculans were abundant. The dominant species in the middle-aged biofilm were Curvularia lunata, Curvularia pallescens, Fusarium oxysporum and Fusarium redolens, and in the young biofilm were Cladosporium cladosporioides, Curvularia clavata, Paraconiothyrium sp. and Phoma eupyrena.

Conclusions: Our results suggest that the composition of the fungal community in each biofilm varies according to time of exposure to the environment. Furthermore, the fungal community was composed of a pool of uncommon species that might be autochthonous to limestone.

Key words: dominant species, fungal colonization, fungal diversity, succession, trophic preference.

\section{Resumen:}

Antecedentes y Objetivos: La colonización de los sustratos líticos por comunidades fúngicas está determinada por las propiedades del sustrato (bioreceptividad) y las condiciones climáticas y microclimáticas. Sin embargo, los efectos del tiempo de exposición de la superficie de la roca calcárea al ambiente sobre la composición de las comunidades fúngicas no se ha investigado. En este estudio, analizamos la composición y estructura de las comunidades fúngicas inmersas en biopelículas asociadas a roca calcárea, en paredes de edificaciones modernas construidas a diferentes tiempos en un ambiente subtropical en Campeche, México.

Métodos: Se consideró una cronosecuencia de paredes construidas a uno, cinco y 10 años. Sobre cada pared, se rasparon tres superficies de $3 \times 3 \mathrm{~cm}$ para cada biopelícula. Los hongos se aislaron por la técnica de lavado y filtración de partículas, posteriormente se inocularon en dos medios de cultivo contrastantes (un medio oligotrófico y uno copiotrófico). Los hongos se identificaron de acuerdo a sus características macro y microscópicas.

Resultados clave: Encontramos 73 géneros y 202 especies de 844 aislados. Los resultados mostraron que las comunidades fúngicas son diferentes en las tres biopelículas. En la biopelícula de desarrollo intermedio encontramos un alto número de aislados, pero tanto la riqueza como la diversidad fueron bajas. En contraste, en la biopelícula avanzada, los valores de riqueza de especies y diversidad fueron altos, y las especies abundantes fueron Hyphomycete 1, Myrothecium roridum y Pestalotiopsis maculans. Las especies dominantes en la biopelícula intermedia fueron Curvularia lunata, Curvularia pallescens, Fusarium oxysporum y Fusarium redolens, y en la biopelícula joven fueron Cladosporium cladosporioides, Curvularia clavata, Paraconiothyrium sp. y Phoma eupyrena.

Conclusiones: Nuestros resultados sugieren que la composición de la comunidad fúngica en cada biopelícula cambia de acuerdo al tiempo de exposición de la roca calcárea al ambiente. Además, como parte de la composición de la comunidad fúngica, encontramos un conjunto de especies poco comunes que podrían ser autóctonas en la roca calcárea.

Palabras clave: colonización fúngica, diversidad fúngica, especies dominantes, preferencia trófica, sucesión. 


\section{INTRODUCTION}

It is well known that rocks, either in natural geological settings or as part of monuments, are common habitats for a wide range of microorganisms (Scheerer et al., 2009; Miller et al., 2012). The colonization of lithic substrates by microbial communities is influenced by the properties of the substrate, such as porosity, surface roughness and mineralogical composition -bioreceptivity- (see review in Miller et al., 2012), in addition to climate and microclimatic conditions (Guillitte, 1995; Ortega-Morales et al., 1999; Gaylarde and Gaylarde, 2005; Barberousse et al., 2006). Furthermore, communities of microorganisms growing on lithic substrates, including fungi, may respond differentially to environmental conditions over time based on their ecophysiological requirements (Scheerer et al., 2009; Mihajlovski et al., 2014).

In the tropics and subtropics, rocks are capable of being colonized by microorganisms due to high levels of relative humidity and particular bioreceptivity of the limestone (Kumar and Kumar, 1999; Gómez-Cornelio et al., 2012). Gaylarde and Gaylarde (2005) found that the macro- and micro-environments of different geographical regions play an important role in the biomass and composition of the microorganism groups that compose biofilms. For example, microbial biomass in Latin America is dominated by cyanobacteria and fungi, while in Europe phototrophs, including algae and cyanobacteria, are the most common organisms. Furthermore, the development of biofilms on rocks represents an important stage in the primary succession of terrestrial ecosystems (Chertov et al., 2004; Gorbushina, 2007). In this process, fungi that form part of biofilms physically and chemically deteriorate rock, and thus actively participate in the formation of protosoil and minerals and also accelerate this process, enabling subsequent colonization of the substrate by mosses, lichens or plants (Gorbushina and Krumbein, 2000; Sterflinger, 2000; Gadd, 2007). Although molecular techniques are commonly used to study communities in the field of environmental microbiology, the traditional techniques of isolation and identification of fungi are of vital importance in order to phenotypically characterize fungi and to determine their role on epilithic substrates (Ruibal et al., 2005; Gleeson et al., 2010).

Fungal epilithic communities have been studied in a wide range of environments and for several lithotypes (Sterflinger and Krumbein, 1997; Sterflinger and Prillinger, 2001; Urzì et al., 2001; Gorbushina et al., 2002; Ruibal et al., 2005; Ruibal et al., 2009; Tang and Lian, 2012). However, most research has not considered the influence of time on the colonization patterns of fungal communities. One exception was the study of Lan et al. (2010), in which fungal communities of young and old biofilms on sandstone were found substantially different. Furthermore, the importance of filamentous fungi as rock colonizers and their ecological role in environments are not well-understood, especially in tropical and subtropical climates. More attention has been placed on the microcolonial fungi, meristematic fungi and yeasts of temperate climates (Sterflinger and Krumbein, 1997; Gorbushina et al., 2002; Chertov et al., 2004; Gorbushina et al., 2005; Ruibal et al., 2005; 2009; Sterflinger et al., 2012). Therefore, in order to expand our current understanding of the fungal community associated with limestone, we studied the culturable subset of fungi in biofilms exposed to similar environmental conditions and substratum properties. A chronosequence was considered by examining the biofilms of three walls constructed one, five and 10 years ago.

\section{MATERIALS AND METHODS}

\section{Study area and climate variables}

The coastal city of Campeche, Mexico has a subtropical climate and an altitudinal range of 3-10 m. The studied biofilms were relatively categorized as young, middleaged and old, corresponding to walls that were constructed with limestone rock fragments one, five and 10 years ago, respectively, according to historical documentation (Fig. 1). The colonization of buildings by fungi may initiate shortly after construction but the formation of biofilm usually takes several years (Barberousse et al., 2006; Gómez-Cornelio et al., 2012; Adamson et al., 2013). Hen- 

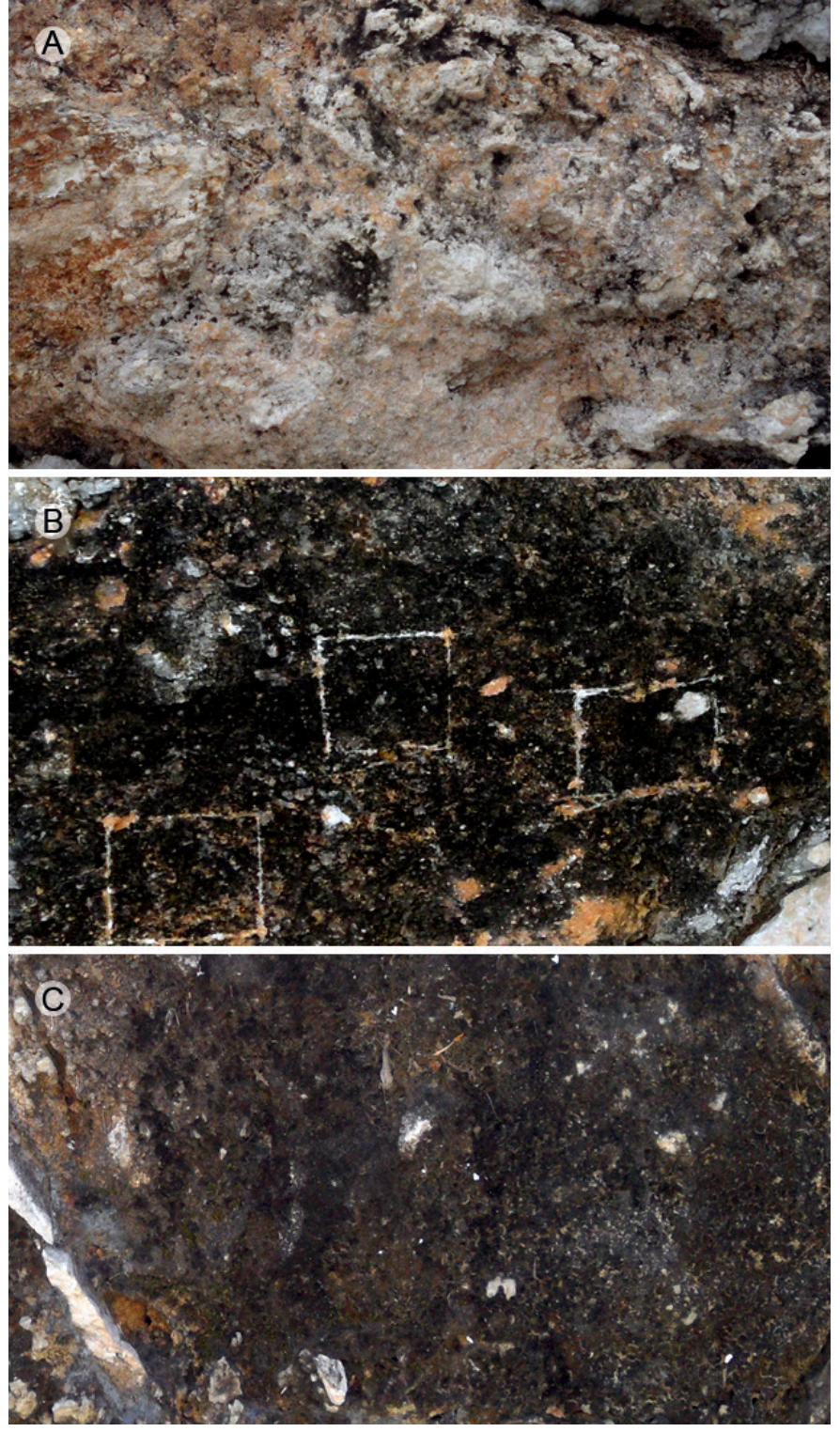

Figure 1: Biofilm samples from rock fragments of limestone buildings (time elapsed since construction): A. young biofilm (one year); B. middle-aged biofilm (five years); C. old biofilm (10 years).

ce, the monthly climate data were obtained from the local meteorological observatory in Campeche, in order to calculate the annual means of climate variables as well as the mean conditions corresponding to the number of years since walls were constructed and exposed to the environment. The considered climatic variables were: minimum and maximum temperature, minimum, mean and maximum relative humidity and mean rainfall (Table 1).
Limestone walls with comparable characteristics of exposure to the surrounding environment were chosen. All walls were composed of rock blocks and had similar substratum properties (or bioreceptivity), vertical surfaces and homogeneous coverage of biofilms. In addition, all walls were oriented towards the north where low solar irradiation and high relative humidity prevail in comparison to facades oriented towards other directions (Adamson et al., 2013; Ortega-Morales et al., 2013). Further criteria for selecting the walls included the absence of surrounding vegetation and low levels of human disturbance, such as painting or washing. Sites with automobile traffic or post-construction remodeling works were avoided. Samples were taken from above the height of one meter in order to avoid confounding factors such as potential microbial colonization due to splashing water. Hence, time elapsed since construction of the walls and establishment of biofilms was the main influential variable considered in the analysis of fungal community structure.

\section{Biofilm sampling and fungal isolation}

In the dry season of January 2014, we sampled biofilms on the three selected limestone surfaces. Mean maximum and minimum temperatures in January were $34{ }^{\circ} \mathrm{C}$ and $11.4{ }^{\circ} \mathrm{C}$, respectively. Mean rainfall was $23 \mathrm{~mm}$, and the mean relative humidity was $83 \%$. On each wall, we scraped three surface areas of $3 \times 3 \mathrm{~cm}$ to a maximum depth of $3 \mathrm{~mm}$, using a sterile scalpel. Scraping was performed by the same person to avoid bias. The biomasses of the biofilms scraped from the wall were placed in sterile Petri dishes and transferred to the laboratory for processing.

Fungi were isolated by washing and filtration of particles technique (Bills et al., 2004). One gram of each scraped biofilm was placed in a washing apparatus with micro-sieves with pores of 250, 125, 100 and 75 $\mu \mathrm{m}$ (MINI-SIEVE INSERT-ASTD, Bel-Art Products, Pequannock, New Jersey, USA), and was consequently washed and filtered for 10 min using bi-distilled water. This technique reduces the isolation of propagules from spores, favoring only the isolation of fungi attached to rock particles (Bills et al., 2004; Arias-Mota and Here- 
Table 1: Geographical location, color and climatic parameters of biofilms developed on the surface of sampled limestone walls. (Values are expressed as means $\pm 1 \mathrm{SD})$.

\begin{tabular}{llccc}
\hline & & Young biofilm & Middle-aged biofilm & Old biofilm \\
\hline Geographical location & & $19^{\circ} 49^{\prime} 30.7^{\prime \prime} \mathrm{N}$ & $19^{\circ} 49^{\prime} 30.5^{\prime \prime} \mathrm{N}$ & $19^{\circ} 49^{\prime} 29.4^{\prime \prime} \mathrm{N}$ \\
& & $90^{\circ} 32^{\prime} 51.2^{\prime \prime} \mathrm{W}$ & $90^{\circ} 33^{\prime} 16.3^{\prime \prime} \mathrm{W}$ & $90^{\circ} 33^{\prime} 14^{\prime \prime} \mathrm{W}$ \\
\hline Mean temperature $\left({ }^{\circ} \mathrm{C}\right)$ & Minimum & $16.5 \pm 2.5$ & $16.4 \pm 4.7$ & $16.7 \pm 2.5$ \\
& Maximum & $37.1 \pm 4.3$ & $37.6 \pm 2.6$ & $37.2 \pm 4.5$ \\
\cline { 2 - 5 } Mean relative humidity (\%) & Minimum & $43.8 \pm 10.7$ & $39.9 \pm 9.4$ & $39.6 \pm 9.4$ \\
& Mean & $78.6 \pm 5.6$ & $75 \pm 5.8$ & $74.5 \pm 6.6$ \\
\multirow{2}{*}{ Mean rainfall (mm) } & Maximum & $97.9 \pm 1.2$ & $97.8 \pm 1.1$ & $97.4 \pm 1.4$ \\
\cline { 2 - 5 } & Rainy season & $224.9 \pm 11.3$ & $192.6 \pm 54$ & $194.8 \pm 29$ \\
\multirow{2}{*}{ Degree of colonization (visual } & Dry season & $20.4 \pm 19$ & $19.8 \pm 17$ & $24.4 \pm 20$ \\
\cline { 2 - 5 } & & Not observed & Dark green & Black \\
\hline
\end{tabular}

dia-Abarca, 2014) that may present active bioweathering or serve a protective function on the surface of the limestone.

Particles trapped on the $75 \mu \mathrm{m}$ sieve were transferred to sterile filter paper and incubated for $24 \mathrm{~h}$ at 27 ${ }^{\circ} \mathrm{C}$ to remove excess water. In order to isolate the greatest number of species, we used two culture media: a copiotrophic medium composed of $2 \%$ malt extract, $2 \%$ agar and $0.2 \% \mathrm{CaCO}_{3}(\mathrm{MEAC})$ and an oligotrophic medium of $0.2 \% \mathrm{CaCO}_{3}$ and $2 \%$ agar (CCOA). Both media were adjusted to $\mathrm{pH} 7.7$ and supplemented with chloramphenicol (200 $\mathrm{mg} \mathrm{L}^{-1}$ ) to inhibit the growth of bacteria. Media were prepared with $\mathrm{CaCO}_{3}$, since it is the main component of limestone (Burford et al., 2003). Under a stereomicroscope, 50 particles were transferred to 10 plates with MEAC ( 5 particles per plate); this procedure was repeated for the CCOA medium. Plates were incubated at $27{ }^{\circ} \mathrm{C}$ in darkness. After the fourth day, plates were inspected daily for a period of four weeks. All fungal colonies that emerged from the particles were purified in inclined tubes with MEAC.

\section{Morphological identification of fungi}

The fungal isolates were identified according to macroscopic characteristics, such as coloration, diameter, textu- re, pigmentation, margin appearance, zonality and production of exudates in the culture medium, in addition to the morphological characteristics of their reproductive and vegetative structures, including color, conidiogenesis, spore type and size. Fungal isolates that sporulated were identified using the taxonomic keys of Booth (1971), Ellis (1971; 1976), Sutton (1980), Pitt (2000), Klich (2002), Boerema et al. (2004), Domsch et al. (2007) and Seifert et al. (2011). The identity of the species with more than eight isolates was confirmed by performing genomic DNA extraction and sequencing the ITS region (data not shown).

Fungal colonies that did not sporulate were inoculated into the following culture media: cornmeal agar, oatmeal agar, potato-carrot agar, Czapek dox agar, potato dextrose agar and V8 agar. Plates were then subjected to cyclical periods of light/darkness $(12 / 12 \mathrm{~h})$ to promote sporulation (Bills et al., 2004) and incubated at $27^{\circ} \mathrm{C}$. Every fourth day for up to six weeks, plates were checked for signs of reproductive structures. Isolates that not produced spores were separated into morphotaxa, according to their macroscopic and microscopic morphology in the different culture media. All fungal isolates were conserved in malt extract broth supplemented with glycerol $(20 \%$ [vol/vol] $)$ at $-80{ }^{\circ} \mathrm{C}$; agar plugs with mycelium were conserved in sterile distilled water at room temperature. 


\section{Data analysis}

The fungal communities of the sampled biofilms were analyzed according to species richness, defined as the number of different fungi species per biofilm, in addition to species abundance as the number of fungal isolates per identified species. The colonization frequency of the particles was determined as number of emerged fungal species (one or two per particle) from particles divided by the number of inoculated particles, multiplied by 100 in order to obtain the percentage of particles with adhered mycelium (Bills et al., 2004). In order to determine the substrates that have been reported for the fungi that were identified at the species level, we used the literature previously employed in the identification of fungi and performed a search in Summon system. Fungal diversity was calculated with the Simpson's (D') and Shannon's (H') diversity indices, in addition to the Shannon (J') evenness index, which were performed in the EstimateS 9.1 software (Colwell, 2013). In order to determine the similarity and composition of the fungal species found in the three biofilms, the Jaccard index was calculated, and a Venn diagram was created.

\section{Results}

\section{Analysis of fungal composition and diversity}

In the mycological analysis 844 isolates were recovered, distributed in 73 genera and 202 species (Table 2). The identified species were grouped as follows: 149 Ascomycota, one Basidiomycota and 52 Mycelia sterilia. Hyphomicetous asexual species of Ascomycota (108 species) dominated, while Coelomycetous species represented $21 \%$ of the isolates ( 38 species). The genera with highest number of species $(>4)$ and isolates $(>17)$ in the fungal community were Aspergillus P. Micheli ex Haller, Cladosporium Link, Curvularia Boedijn, Fusarium Link, Microsphaeropsis Höhn., Myrothecium Tode, Nodulisporium Preuss, Paraconiothyrium Verkley and Phoma Sacc. (Table 2).

We found the largest number of isolates (322) in the middle-aged biofilm, followed by the old and young biofilms with 268 and 254 isolates, respectively. However, species richness and diversity were higher in the old biofilm and lower in the middle-aged biofilm (Table 3). The Shannon evenness index of the old biofilm generated a value close to 1, and in the middle-aged biofilm, a value of 0.47 (Table 3). The Jaccard's similarity index showed a low degree of similarity among the young, middle-aged and old fungal communities inhabiting the biofilms. The resulting index values were similar for the comparisons of young and middle-aged (0.18), young and old (0.16) and middle-aged and old (0.15) biofilms.

Overall, of the 202 species identified, 26\% were isolated from inoculated particles in both culture media (52 species), while 36\% (73 species) were found exclusively in the oligotrophic medium (CCOA) and 38\% in the copiotrophic medium (MEAC). Additionally, in the analysis of colonization frequency of particles from all three biofilms, we observed a high number of particles with adhered mycelia (Table 3). The middle-aged biofilm showed the highest percentage $(80 \%)$ of colonization; however, most mycelium that emerged from these particles belonged to the species Curvularia lunata (Wakker) Boedijn, Fusarium oxysporum Schltdl. and Fusarium redolens Wollenw. (Table 2). The young and old biofilms presented a minor colonization frequency of particles (Table 3).

In the old biofilm, a high number of isolated species (35\%) was specific to only one of the media, and a slightly lower proportion was present in both media (30\%). In young and middle-aged biofilms, $41 \%$ of species were indistinctly isolated from both media (Table 2). Isolates of Lasiodiplodia theobromae (Pat.) Griffon \& Maubl. and Nigrospora oryzae (Berk. \& Broome) Petch were obtained in the MEAC medium. A high percentage (>70\%) of species from the genera Aspergillus, Penicillium Link and Trichoderma Pers. were also isolated. Meanwhile, in the CCOA medium the lithic species Friedmanniomyces simplex Selbmann, de Hoog, Mazzaglia, Friedmann \& Onofri (6 isolates) was found as well as Stachybotrys Corda species and a large number of uncommon species (Table 2). 
Table 2: Epilithic fungal community in terms of abundance of species isolated from biofilms on limestone at different stages of development and color of their reproductive structures. (M: melanized and $\mathrm{H}$ : hyaline).

${ }^{\mathrm{a}}$ Copiotrophic medium (MEAC).

bligotrophic medium (CCOA).

*Mycelia sterilia with one or more isolates, which are added in the total.

\begin{tabular}{|c|c|c|c|c|c|}
\hline Epilithic fungi & Coloration & Young biofilm & $\begin{array}{l}\text { Middle-aged } \\
\text { biofilm }\end{array}$ & Old biofilm & Total \\
\hline \multicolumn{6}{|l|}{ Ascomycota } \\
\hline Sordaria fimicola (Roberge ex Desm.) Ces. \& De Not. & M & & 2 & 1 & $3^{\mathrm{a}, \mathrm{b}}$ \\
\hline Xylariales sp. 1 & M & & & 1 & $1^{\mathrm{b}}$ \\
\hline Xylariales sp. 2 & M & & & 1 & $1^{\mathrm{a}}$ \\
\hline Xylariales sp. 3 & M & & & 1 & $1^{\mathrm{b}}$ \\
\hline \multicolumn{6}{|l|}{ Coelomycetous asexual species of Ascomycota } \\
\hline Ascochyta carpathica (Allesch.) Keissl. & M & & & 2 & $2^{\mathrm{a}}$ \\
\hline Clypeopycnis sp. & M & 2 & 1 & 3 & $6^{\mathrm{a}, \mathrm{b}}$ \\
\hline Coleophoma sp. & M & 7 & & & $7^{\mathrm{a}, \mathrm{b}}$ \\
\hline Colletotrichum crassipes (Speg.) Arx & $\mathrm{H}$ & 1 & & & $1^{\mathrm{a}}$ \\
\hline Colletotrichum dematium (Pers.) Grove & M & 1 & & & $1^{\mathrm{a}}$ \\
\hline Colletotrichum gloeosporioides (Penz.) Penz. \& Sacc. & $\mathrm{H}$ & 1 & & & $1^{b}$ \\
\hline $\begin{array}{l}\text { Coniothyrium multiporum (V.H. Pawar, P.N. Mathur \& } \\
\text { Thirum.) Verkley \& Gruyter }\end{array}$ & M & & 1 & & $1^{b}$ \\
\hline Cytospora polygoni-sieboldii Henn. & M & & & 1 & $1^{\mathrm{a}}$ \\
\hline Lasiodiplodia theobromae (Pat.) Griffon \& Maubl. & M & & & 7 & $7^{\mathrm{a}}$ \\
\hline Microsphaeropsis arundinis (S. Ahmad) B. Sutton & M & & 1 & 2 & $3^{\mathrm{a}, \mathrm{b}}$ \\
\hline Microsphaeropsis sp. 1 & M & & & 1 & $1^{\mathrm{b}}$ \\
\hline Microsphaeropsis sp. 2 & M & & 2 & & $2^{\mathrm{a}, \mathrm{b}}$ \\
\hline $\begin{array}{l}\text { Neosetophoma samararum (Desm.) Gruyter, Aveskamp \& } \\
\text { Verkley }\end{array}$ & M & & & 1 & $1^{\mathrm{a}}$ \\
\hline Paraconiothyrium sp. & M & 19 & 3 & & $22^{\mathrm{a}, \mathrm{b}}$ \\
\hline $\begin{array}{l}\text { Paraphoma chrysanthemicola (Hollós) Gruyter, Aveskamp } \\
\text { \& Verkley }\end{array}$ & M & 1 & & & $1^{\mathrm{b}}$ \\
\hline Paraphoma fimeti (Brunaud) Gruyter, Aveskamp \& Verkley & M & 2 & & 3 & $5^{\mathrm{a}, \mathrm{b}}$ \\
\hline Pestalotiopsis maculans (Corda) Nag Raj & M & 5 & 3 & 10 & $18^{\mathrm{a}, \mathrm{b}}$ \\
\hline $\begin{array}{l}\text { Peyronellaea aurea (Gruyter, Noordel. \& Boerema) } \\
\text { Aveskamp, Gruyter \& Verkley }\end{array}$ & M & 2 & & & $2^{\mathrm{a}, \mathrm{b}}$ \\
\hline $\begin{array}{l}\text { Peyronellaea gardeniae (S. Chandra \& Tandon) Aveskamp, } \\
\text { Gruyter \& Verkley }\end{array}$ & M & 1 & & & $1^{\mathrm{b}}$ \\
\hline Phlyctema lappae (P. Karst.) Sacc. & M & 1 & & & $1^{\mathrm{a}}$ \\
\hline Phoma adianticola (E. Young) Boerema & M & 4 & & & $4^{\mathrm{a}}$ \\
\hline Phoma crystallifera Gruyter, Noordel. \& Boerema & M & & & 1 & $1^{\mathrm{b}}$ \\
\hline Phoma eupyrena Sacc. & M & 23 & 12 & 15 & $50^{\mathrm{a}, \mathrm{b}}$ \\
\hline
\end{tabular}


Table 2: Continuation.

\begin{tabular}{|c|c|c|c|c|c|}
\hline Epilithic fungi & Coloration & Young biofilm & $\begin{array}{l}\text { Middle-aged } \\
\text { biofilm }\end{array}$ & Old biofilm & Total \\
\hline Phoma herbarum Westend. & $\mathrm{M}$ & 4 & 1 & 2 & $7^{\mathrm{a}, \mathrm{b}}$ \\
\hline Phoma heteroderae Sen Y. Chen, D.W. Dicks. \& Kimbr. & M & 2 & & & $2^{\mathrm{a}, \mathrm{b}}$ \\
\hline Phoma leveillei Boerema \& G.J. Bollen & M & & & 1 & $1^{\mathrm{b}}$ \\
\hline $\begin{array}{l}\text { Phoma multirostrata (P.N. Mathur, S.K. Menon \& Thirum.) } \\
\text { Dorenb. \& Boerema }\end{array}$ & M & & 1 & 1 & $2^{\mathrm{b}}$ \\
\hline Phoma paspali P.R. Johnst. & M & 5 & & & $5^{\mathrm{a}, \mathrm{b}}$ \\
\hline Phoma pratorum P.R. Johnst. \& Boerema & M & & 1 & & $1^{\mathrm{a}}$ \\
\hline Phoma proteae Crous & M & & & 1 & $1^{\mathrm{b}}$ \\
\hline Phoma putamina Speg. & M & 1 & & 2 & $3^{\mathrm{a}, \mathrm{b}}$ \\
\hline Phoma sp. 1 & M & 1 & & & $1^{\mathrm{a}}$ \\
\hline Phoma sp. 2 & M & 2 & & & $2^{\mathrm{a}, \mathrm{b}}$ \\
\hline Phoma tropica R. Schneid. \& Boerema & M & & 2 & & $2^{\mathrm{a}, \mathrm{b}}$ \\
\hline Phomopsis putator (Nitschke) Traverso & M & & & 1 & $1^{\mathrm{a}}$ \\
\hline Pleurophomopsis lignicola Petr. & M & 1 & 1 & & $2^{\mathrm{a}}$ \\
\hline $\begin{array}{l}\text { Pyrenochaetopsis pratorum (Berk. \& M.A. Curtis) M.B. } \\
\text { Ellis }\end{array}$ & M & 1 & & & $1^{\mathrm{b}}$ \\
\hline $\begin{array}{l}\text { Westerdykella minutispora (P.N. Mathur) Gruyter, Aveskamp } \\
\text { \& Verkley }\end{array}$ & M & 4 & 1 & 1 & $6^{\mathrm{a}, \mathrm{b}}$ \\
\hline \multicolumn{6}{|l|}{ Hyphomicetous asexual species of Ascomycota } \\
\hline Acremoniella velutina (Fuckel) Sacc. & M & 1 & & & $1^{\mathrm{a}}$ \\
\hline Acremonium brachypenium $\mathrm{W}$. Gams & $\mathrm{H}$ & & 2 & & $2^{\mathrm{b}}$ \\
\hline Acremonium fusidioides (Nicot) W. Gams & $\mathrm{H}$ & & & 1 & $1^{\mathrm{b}}$ \\
\hline Acremonium rutilum W. Gams & $\mathrm{H}$ & & & 2 & $2^{\mathrm{b}}$ \\
\hline Acremonium sordidulum W. Gams \& D. Hawksw. & $\mathrm{H}$ & & & 1 & $1^{\mathrm{a}}$ \\
\hline Agaricodochium sp. & $\mathrm{H}$ & 1 & & & $1^{\mathrm{a}}$ \\
\hline Alternaria longipes (Ellis \& Everh.) E.W. Mason & M & & & 1 & $1^{\mathrm{a}}$ \\
\hline Alternaria tenuissima (Kunze) Wiltshire & M & 1 & 1 & & $2^{\mathrm{a}, \mathrm{b}}$ \\
\hline Arxiella terrestris Papendorf & $\mathrm{H}$ & & & 1 & $1^{\mathrm{b}}$ \\
\hline Aspergillus aculeatus Iizuka & M & & & 2 & $2^{\mathrm{a}}$ \\
\hline Aspergillus alliaceus Thom \& Church & $\mathrm{H}$ & & & 1 & $1^{\mathrm{a}}$ \\
\hline Aspergillus awamori Nakaz. & M & & & 2 & $2^{\mathrm{a}}$ \\
\hline Aspergillus foetidus Thom \& Raper & M & & & 2 & $2^{\mathrm{a}}$ \\
\hline Aspergillus fumigatus Fresen. & M & & & 2 & $2^{\mathrm{a}}$ \\
\hline Aspergillus japonicus Saito & M & & & 1 & $1^{\mathrm{a}}$ \\
\hline Aspergillus niger Tiegh. & M & & & 7 & $7^{\mathrm{a}}$ \\
\hline Aureobasidium pullulans (de Bary \& Löwenthal) G. Arnaud & M & & & 1 & $1^{\mathrm{a}}$ \\
\hline Badarisama sp. & M & & 1 & & $1^{\mathrm{a}}$ \\
\hline
\end{tabular}


Table 2: Continuation.

\begin{tabular}{|c|c|c|c|c|c|}
\hline Epilithic fungi & Coloration & Young biofilm & $\begin{array}{l}\text { Middle-aged } \\
\text { biofilm }\end{array}$ & Old biofilm & Total \\
\hline Baudoinia sp. & $\mathrm{M}$ & & & 1 & $1^{\mathrm{b}}$ \\
\hline Calcarisporium sp. & M & 1 & & & $1^{\mathrm{a}}$ \\
\hline Capnobotryella antalyensis Sert \& Sterfl. & M & & & 1 & $1^{\mathrm{a}}$ \\
\hline Chaetasbolisia falcata V.A.M. Mill. \& Bonar & M & 1 & & & $1^{\mathrm{b}}$ \\
\hline Chromelosporium sp. & $\mathrm{H}$ & & & 1 & $1^{\mathrm{a}}$ \\
\hline Cladosporium cladosporioides (Fresen.) G.A. de Vries & M & 22 & 10 & 8 & $40^{\mathrm{a}, \mathrm{b}}$ \\
\hline Cladosporium oxysporum Berk. \& M.A. Curtis & $\mathrm{M}$ & 10 & & 8 & $18^{\mathrm{a}, \mathrm{b}}$ \\
\hline Cladosporium sphaerospermum Penz. & M & 9 & & 2 & $11^{\mathrm{a}, \mathrm{b}}$ \\
\hline Cladosporium tenuissimum Cooke & M & 2 & & 2 & $4^{\mathrm{a}, \mathrm{b}}$ \\
\hline Corynespora citricola M.B. Ellis & M & 1 & & & $1^{\mathrm{a}}$ \\
\hline Corynesporella pinarensis R.F. Castañeda & M & & & 1 & $1^{\mathrm{b}}$ \\
\hline $\begin{array}{l}\text { Curvularia australiensis (Tsuda \& Ueyama) Manamgoda, L. } \\
\text { Cai \& K.D. Hyde }\end{array}$ & M & 1 & 1 & 5 & $7^{\mathrm{a}, \mathrm{b}}$ \\
\hline Curvularia brachyspora Boedijn & M & & 2 & & $2^{\mathrm{a}}$ \\
\hline Curvularia clavata B.L. Jain & M & 18 & 3 & 1 & $22^{\mathrm{a}, \mathrm{b}}$ \\
\hline Curvularia fallax Boedijn & M & 1 & & & $1^{\mathrm{b}}$ \\
\hline $\begin{array}{l}\text { Curvularia hawaiiensis (Bugnic. ex M.B. Ellis) Manamgoda, } \\
\text { L. Cai \& K.D. Hyde }\end{array}$ & M & & 1 & & $1^{\mathrm{b}}$ \\
\hline Curvularia lunata (Wakker) Boedijn & M & 10 & 105 & 26 & $141^{\mathrm{a}, \mathrm{b}}$ \\
\hline Curvularia pallescens Boedijn & M & 6 & 13 & 11 & $30^{\mathrm{a}, \mathrm{b}}$ \\
\hline Curvularia spicifera (Bainier) Boedijn & M & 1 & & & $1^{\mathrm{a}}$ \\
\hline Curvularia verruculosa Tandon \& Bilgrami ex. M.B. Ellis & M & & 7 & 1 & $8^{\mathrm{a}, \mathrm{b}}$ \\
\hline Curvularia sp. & M & & & 2 & $2^{\mathrm{a}, \mathrm{b}}$ \\
\hline Echinocatena sp. & M & & & 1 & $1^{\mathrm{b}}$ \\
\hline Exochalara longissima (Grove) W. Gams \& Hol.-Jech. & M & & & 2 & $2^{\mathrm{a}}$ \\
\hline $\begin{array}{l}\text { Friedmanniomyces simplex Selbmann, de Hoog, Mazzaglia, } \\
\text { Friedmann \& Onofri }\end{array}$ & M & 1 & 3 & 2 & $6^{\mathrm{b}}$ \\
\hline Fusarium camptoceras Wollenw. \& Reinking & $\mathrm{H}$ & 1 & & & $1^{\mathrm{b}}$ \\
\hline Fusarium equiseti (Corda) Sacc. & $\mathrm{H}$ & 1 & & & $1^{\mathrm{a}}$ \\
\hline Fusarium flocciferum Corda & $\mathrm{H}$ & 1 & 1 & & $2^{\mathrm{a}, \mathrm{b}}$ \\
\hline Fusarium incarnatum (Desm.) Sacc. & $\mathrm{H}$ & & & 1 & $1^{\mathrm{b}}$ \\
\hline Fusarium oxysporum Schltdl. & $\mathrm{H}$ & 8 & 40 & 4 & $52^{\mathrm{a}, \mathrm{b}}$ \\
\hline Fusarium redolens Wollenw. & $\mathrm{H}$ & 14 & 41 & 9 & $64^{\mathrm{a}, \mathrm{b}}$ \\
\hline Fusarium sacchari (E.J. Butler \& Hafiz Khan) W. Gams & $\mathrm{H}$ & 1 & & & $1^{\mathrm{a}}$ \\
\hline Fusarium solani (Mart.) Sacc. & $\mathrm{H}$ & 2 & & 2 & $4^{\mathrm{a}, \mathrm{b}}$ \\
\hline $\begin{array}{l}\text { Fusarium subglutinans (Wollenw. \& Reinking) P.E. Nelson, } \\
\text { Toussoun \& Marasas }\end{array}$ & $\mathrm{H}$ & 1 & & & $1^{\mathrm{b}}$ \\
\hline
\end{tabular}


Table 2: Continuation.

\begin{tabular}{|c|c|c|c|c|c|}
\hline Epilithic fungi & Coloration & Young biofilm & $\begin{array}{c}\text { Middle-aged } \\
\text { biofilm }\end{array}$ & Old biofilm & Total \\
\hline Fusarium tabacinum (J.F.H. Beyma) W. Gams & $\mathrm{H}$ & & 1 & & $1^{\mathrm{b}}$ \\
\hline Fusarium ventricosum Appel \& Wollenw. & $\mathrm{H}$ & 1 & & & $1^{\mathrm{a}}$ \\
\hline Gabarnaudia sp. & $\mathrm{H}$ & & & 1 & $1^{\mathrm{b}}$ \\
\hline Geotrichum candidum Link & $\mathrm{H}$ & & & 2 & $2^{\mathrm{a}, \mathrm{b}}$ \\
\hline Gilmaniella subornata Morinaga, Minoura \& Udagawa & M & & 1 & & $1^{\mathrm{a}}$ \\
\hline Graphium penicillioides Corda & M & & & 2 & $2^{\mathrm{a}, \mathrm{b}}$ \\
\hline Hyphomycete 1 & M & & & 11 & $11 \mathrm{a}, \mathrm{b}$ \\
\hline Microdochium dimerum (Penz.) Arx & $\mathrm{H}$ & & 1 & 2 & $3^{b}$ \\
\hline Microdochium nivale (Fr.) Samuels \& I.C. Hallett & $\mathrm{H}$ & 2 & & & $2^{\mathrm{b}}$ \\
\hline Monodictys fluctuata (Tandon \& Bilgrami) M.B. Ellis & M & 1 & & & $1^{\mathrm{b}}$ \\
\hline Monodictys paradoxa (Corda) S. Hughes & M & 3 & & & $3^{\mathrm{a}, \mathrm{b}}$ \\
\hline Myrothecium cinctum (Corda) Sacc. & M & 3 & & & $3^{\mathrm{a}}$ \\
\hline Myrothecium roridum Tode & M & & 12 & 16 & $28^{\mathrm{a}, \mathrm{b}}$ \\
\hline Myrothecium sp. 1 & M & 1 & 3 & & $4^{\mathrm{a}, \mathrm{b}}$ \\
\hline Myrothecium sp. 2 & M & & 1 & & $1^{\mathrm{a}}$ \\
\hline Myrothecium sp. 3 & M & & 1 & & $1^{\mathrm{a}}$ \\
\hline Nalanthamala madreeya Subram. & $\mathrm{H}$ & & 1 & & $1^{\mathrm{b}}$ \\
\hline Nigrospora oryzae (Berk. \& Broome) Petch & M & 3 & 1 & 1 & $5^{\mathrm{a}}$ \\
\hline Nodulisporium acervatum (Massee) Deighton & M & & & 1 & $1^{\mathrm{b}}$ \\
\hline Nodulisporium ochraceum Preuss & M & 1 & & & $1^{\mathrm{a}}$ \\
\hline Nodulisporium puniceum (Cooke \& Ellis) Deighton & M & 3 & & 2 & $5^{\mathrm{a}, \mathrm{b}}$ \\
\hline Nodulisporium radians (Berk.) Deighton & M & & & 1 & $1^{\mathrm{b}}$ \\
\hline Nodulisporium sp. 1 & M & 1 & & & $1^{\mathrm{b}}$ \\
\hline Nodulisporium sp. 2 & M & & & 1 & $1^{\mathrm{a}}$ \\
\hline Nodulisporium sylviforme Deighton & M & 1 & & 4 & $5^{\mathrm{a}, \mathrm{b}}$ \\
\hline Nodulisporium thelenum (Sacc.) G. Sm. & M & 1 & & 1 & $2^{a, b}$ \\
\hline $\begin{array}{l}\text { Ochroconis tshawytschae (Doty \& D.W. Slater) Kiril. \& } \\
\text { Al-Achmed }\end{array}$ & M & & & 1 & $1^{\mathrm{b}}$ \\
\hline Penicillium citreonigrum Dierckx & $\mathrm{H}$ & & & 1 & $1^{\mathrm{a}}$ \\
\hline Penicillium dierckxii Biourge & $\mathrm{H}$ & & & 1 & $1^{\mathrm{b}}$ \\
\hline Penicillium islandicum Sopp & $\mathrm{H}$ & & & 1 & $1^{\mathrm{a}}$ \\
\hline Penicillium oxalicum Currie \& Thom & $\mathrm{H}$ & & & 1 & $1^{\mathrm{a}}$ \\
\hline Periconia igniaria E.W. Mason \& M.B. Ellis & M & 1 & & & $1^{\mathrm{a}}$ \\
\hline Periconiella mucunae M.B. Ellis & M & & & 1 & $1^{\mathrm{a}}$ \\
\hline $\begin{array}{l}\text { Prathoda longissima (Deighton \& MacGarvie) E.G. } \\
\text { Simmons }\end{array}$ & M & 1 & & & $1^{\mathrm{b}}$ \\
\hline
\end{tabular}


Table 2: Continuation.

\begin{tabular}{|c|c|c|c|c|c|}
\hline Epilithic fungi & Coloration & Young biofilm & $\begin{array}{c}\text { Middle-aged } \\
\text { biofilm }\end{array}$ & Old biofilm & Total \\
\hline Pseudohelicomyces albus Garnica \& E. Valenz. & $\mathrm{H}$ & & & 2 & $2^{\mathrm{a}, \mathrm{b}}$ \\
\hline $\begin{array}{l}\text { Pseudopithomyces chartarum (Berk. \& M.A. Curtis) J.F. Li, } \\
\text { Ariyawansa \& K.D. Hyde }\end{array}$ & M & 1 & & & $1^{\mathrm{a}}$ \\
\hline $\begin{array}{l}\text { Pseudoramichloridium brasilianum (Arzanlou \& Crous) } \\
\text { Cheew. \& Crous }\end{array}$ & M & & 1 & & $1^{\mathrm{a}}$ \\
\hline $\begin{array}{l}\text { Ramichloridium apiculatum (J.H. Mill., Giddens \& A.A. } \\
\text { Foster) de Hoog }\end{array}$ & M & & 2 & & $2^{\mathrm{b}}$ \\
\hline Sarocladium kiliense (Grütz) Summerb. & $\mathrm{H}$ & & 1 & & $1^{\mathrm{a}}$ \\
\hline Sarocladium strictum (W. Gams) Summerb. & $\mathrm{H}$ & 1 & & & $1^{\mathrm{b}}$ \\
\hline Scolecobasidium constrictum E.V. Abbott & M & 3 & 4 & 1 & $8^{\mathrm{a}, \mathrm{b}}$ \\
\hline Sepedonium sp. & $\mathrm{H}$ & & & 1 & $1^{\mathrm{b}}$ \\
\hline $\begin{array}{l}\text { Stachybotrys microspora (B.L. Mathur \& Sankhla) S.C. Jong } \\
\text { \& E.E. Davis }\end{array}$ & M & & & 1 & $1^{\mathrm{b}}$ \\
\hline Stachybotrys nephrospora Hansf. & M & & & 1 & $1^{\mathrm{b}}$ \\
\hline Stachybotrys renispora P.C. Misra & M & & & 1 & $1^{\mathrm{b}}$ \\
\hline Tolypocladium sp. & $\mathrm{H}$ & 1 & & & $1^{\mathrm{b}}$ \\
\hline Torula herbarum (Pers.) Link & M & & & 1 & $1^{\mathrm{a}}$ \\
\hline Trichobotrys sp. & M & & 1 & & $1^{\mathrm{a}}$ \\
\hline Trichocladium sp. & M & & 1 & & $1^{\mathrm{b}}$ \\
\hline Trichoderma aggressivum Samuels \& W. Gams & $\mathrm{H}$ & 1 & & 1 & $2^{\mathrm{a}}$ \\
\hline Trichoderma harzianum Rifai & $\mathrm{H}$ & & 2 & 1 & $3^{\mathrm{a}, \mathrm{b}}$ \\
\hline Trichoderma longibrachiatum Rifai & $\mathrm{H}$ & & & 2 & $2^{\mathrm{a}}$ \\
\hline Trichoderma ovalisporum Samuels \& Schroers & $\mathrm{H}$ & & & 1 & $1^{\mathrm{a}}$ \\
\hline Trichoderma strigosum Bissett & $\mathrm{H}$ & & & 1 & $1^{\mathrm{a}}$ \\
\hline Veronaea musae M.B. Ellis & M & & 1 & & $1^{\mathrm{a}}$ \\
\hline $\begin{array}{l}\text { Verruconis verruculosa (R.Y. Roy, R.S. Dwivedi \& R.R. } \\
\text { Mishra) Samerp. \& de Hoog }\end{array}$ & M & & 1 & & $1^{\mathrm{a}}$ \\
\hline \multicolumn{6}{|l|}{ Basidiomycota } \\
\hline Geotrichopsis sp. & $\mathrm{H}$ & & & 1 & $1^{\mathrm{a}}$ \\
\hline \multicolumn{6}{|l|}{ Mycelia sterilia } \\
\hline Mycelia sterilia (Morphotaxon 01) & $\mathrm{H}$ & 1 & 1 & 1 & $3^{\mathrm{b}}$ \\
\hline Mycelia sterilia (Morphotaxon 02) & M & 2 & & & $2^{\mathrm{a}, \mathrm{b}}$ \\
\hline Mycelia sterilia (Morphotaxon 03) & $\mathrm{H}$ & 1 & 1 & & $2^{\mathrm{b}}$ \\
\hline Mycelia sterilia (Morphotaxon 04) & M & 1 & & 1 & $2^{b}$ \\
\hline Mycelia sterilia (Morphotaxon 05) & M & 1 & & 1 & $2^{\mathrm{a}, \mathrm{b}}$ \\
\hline Mycelia sterilia (Morphotaxon 06-10*) & M & 1 & & & $5^{\mathrm{a}}$ \\
\hline Mycelia sterilia (Morphotaxon 11-15*) & M & 1 & & & $5^{\mathrm{b}}$ \\
\hline Mycelia sterilia (Morphotaxon 16) & $\mathrm{H}$ & 1 & & & $1^{\mathrm{b}}$ \\
\hline
\end{tabular}


Table 2: Continuation.

\begin{tabular}{|c|c|c|c|c|c|}
\hline Epilithic fungi & Coloration & Young biofilm & $\begin{array}{c}\text { Middle-aged } \\
\text { biofilm }\end{array}$ & Old biofilm & Total \\
\hline 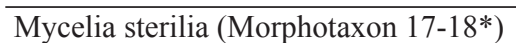 & $\mathrm{M}$ & & 3 & & $6^{\mathrm{a}, \mathrm{b}}$ \\
\hline Mycelia sterilia (Morphotaxon 19-20*) & M & & 2 & & $4^{a, b}$ \\
\hline Mycelia sterilia (Morphotaxon 21) & M & & 2 & & $2^{\mathrm{b}}$ \\
\hline Mycelia sterilia (Morphotaxon 22-23*) & M & & 1 & & $2^{\mathrm{a}}$ \\
\hline Mycelia sterilia (Morphotaxon $24-25^{*}$ ) & $\mathrm{H}$ & & 1 & & $2^{\mathrm{b}}$ \\
\hline Mycelia sterilia (Morphotaxon 26) & M & & 1 & & $1^{\mathrm{b}}$ \\
\hline Mycelia sterilia (Morphotaxon 27) & $\mathrm{H}$ & & & 3 & $3^{\mathrm{a}, \mathrm{b}}$ \\
\hline Mycelia sterilia (Morphotaxon 28) & M & & & 2 & $2^{\mathrm{b}}$ \\
\hline Mycelia sterilia (Morphotaxon 29-34*) & M & & & 1 & $6^{\mathrm{a}}$ \\
\hline Mycelia sterilia (Morphotaxon 35-39*) & $\mathrm{H}$ & & & 1 & $5^{\mathrm{a}}$ \\
\hline Mycelia sterilia (Morphotaxon $40-50^{*}$ ) & M & & & 1 & $11^{\mathrm{b}}$ \\
\hline Mycelia sterilia (Morphotaxon 51-52*) & $\mathrm{H}$ & & & 1 & $2^{\mathrm{b}}$ \\
\hline
\end{tabular}

Table 3: Abundance, richness, diversity and evenness of the fungal epilithic community colonizing biofilms on limestone at different stages of development.

\begin{tabular}{lcccccc}
\hline State of biofilms & $\begin{array}{c}\text { Abundance } \\
\text { (number of } \\
\text { isolates) }\end{array}$ & $\begin{array}{c}\text { Richness (number } \\
\text { of species) }\end{array}$ & $\begin{array}{c}\text { Simpson Diversity } \\
\text { index (D') }\end{array}$ & $\begin{array}{c}\text { Shannon Diversity } \\
\text { index (H') }\end{array}$ & $\begin{array}{c}\text { Shannon evenness } \\
\text { index (J') }\end{array}$ & $\begin{array}{c}\text { Particles } \\
\text { colonization (\%) }\end{array}$ \\
\hline Young & 254 & 83 & 25.8 & 3.7 & 0.67 & 73 \\
Middle-aged & 322 & 59 & 6.8 & 2.7 & 0.47 & 86 \\
Old & 268 & 117 & 37.2 & 4.1 & 0.75 & 75 \\
\hline
\end{tabular}

Of the 124 fungi identified at the species level, many were associated with numerous substrates, based on the literature review to determine with which substrates identified fungal species had been previously associated (Fig. 2). Thirty-one species were identified as cosmopolitan and belonged to the genera Cladosporium, Curvularia, Fusarium and Penicillium, including several common species, such as Aureobasidium pullulans (de Bary \& Löwenthal) G. Arnaud and Geotrichum candidum Link (Domsch et al., 2007). Most of the identified species (81) have been reported in soil, mainly species of the genera Aspergillus, Microdochium Syd., Monodictys S. Hughes, Myrothecium, Phoma, Scolecobasidium E.V. Abbott and Trichoderma. Many species (78) were also associated with plants, including the genera Alternaria, Colletotrichum Corda, Microsphaeropsis, Monodictys, Myrothecium, Phoma and Sarocladium W. Gams \& D. Hawksw. 44 of the identified species have been found in litter, corresponding to the genera of Myrothecium, Scolecobasidium and Stachybotrys. Forty species (genus Monodictys) have been associated with air, and 39 species with wood (genera Nodulisporium and Phlyctema 


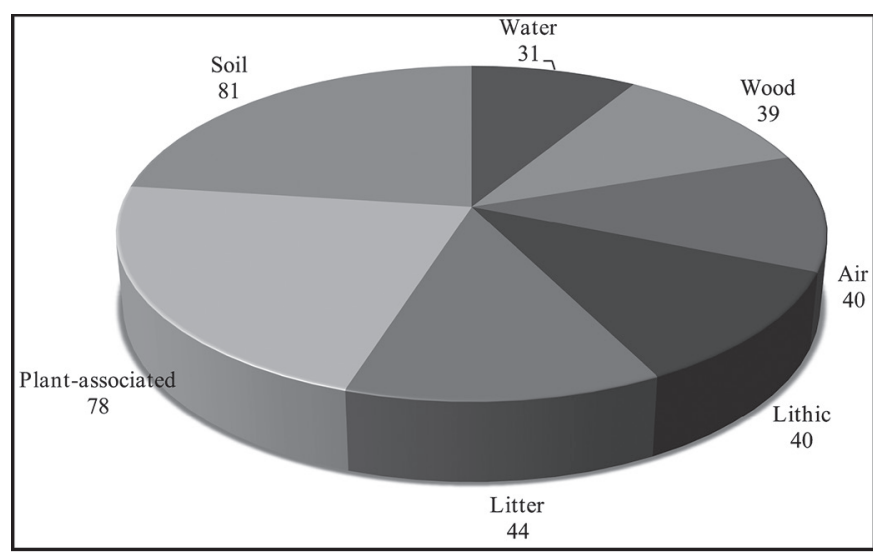

Figure 2: Frequency of substrate types reported for the fungal species isolated from biofilms according to the literature.

Desm.). Finally, 40 and 31 species have been reported on rocks and in water, respectively.

\section{Species dominance}

With respect to species abundance, $61 \%$ of all fungal species were isolated only once, and $6 \%$ were isolated more than 10 times. The remaining fungi $(33 \%)$ were isolated from 2 to 9 times. The encountered fungal community was mainly dominated by fungi that contain melanin at some or all of their reproductive stages (149 species). Only 53 species $(26 \%)$ were found with hyaline structures without pigments (Table 2). In all biofilms, we found that species composition has an approximate ratio of $4: 1$ of melanized fungi to hyaline species.

In regard to the fungal communities, most co-existing groups of species may be associated with a particular biofilm, characterized by time of exposure of the substrate (limestone) to the environment; this is shown in the Venn diagram (Fig. 3). In the young biofilm, we found 49 exclusive species, including several from the genera Colletotrichum and Coleophoma Höhn.; the species Monodyctis paradoxa (Corda) S. Hughes, Myrothecium cinctum (Corda) Sacc., Phoma adianticola (E. Young) Boerema and Phoma paspali P.R. Johnst. were also prominent. In the middle-aged biofilm, only 32 exclusive species were found, although these were not frequent ( $>2$ isolates). The

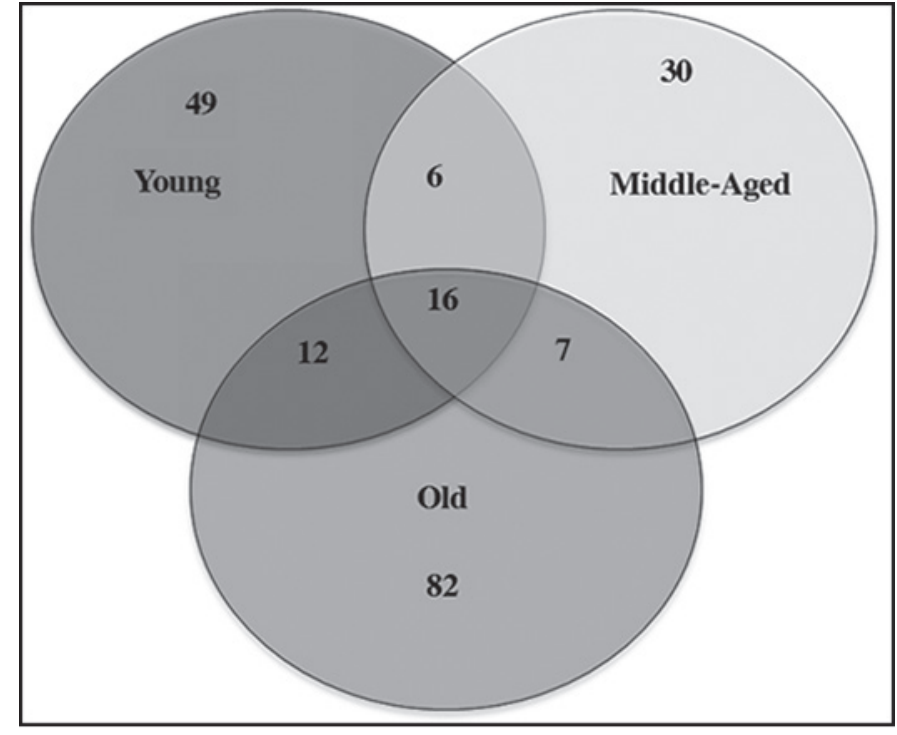

Figure 3: Venn diagram indicating number of common and exclusive fungal species to the studied biofilms.

old biofilms had the highest number of exclusive species (82), among these species of the genera Aspergillus, Lasiodiplodia Ellis \& Everh., Penicillium and Stachybotrys, in addition to Xylariales spp. and Hyphomycete 1.

The Venn diagram shows 16 species in the core group (three biofilms) of the fungal community (Fig. 3 and Table 2). Meanwhile, Cladosporium cladosporioides (Fresen.) G.A. de Vries, Curvularia clavata B.L. Jain, Nigrospora oryzae, Phoma eupyrena Sacc., Phoma herbarum Westend. and Westerdykella minutispora (P.N. Mathur) Gruyter, Aveskamp \& Verkley were abundant in the young biofilm. In the middle-aged biofilm Curvularia lunata, Curvularia pallescens Boedijn, Friedmanniomyces simplex, Fusarium oxysporum, Fusarium redolens and Scolecobasidium constrictum E.V. Abbott, and in the old biofilm Clypeopycnis sp., Curvularia australiensis (Tsuda \& Ueyama) Manamgoda, L. Cai \& K.D. Hyde and Pestalotiopsis maculans (Corda) Nag Raj were frequent (Fig. 4 and Table 2). Twenty-five species were present in at least two biofilms; the young and old biofilms had the highest number of shared species (12). Paraconiothyrium sp. was dominant and was isolated 19 times in the young biofilm, although its abundance diminished in the 


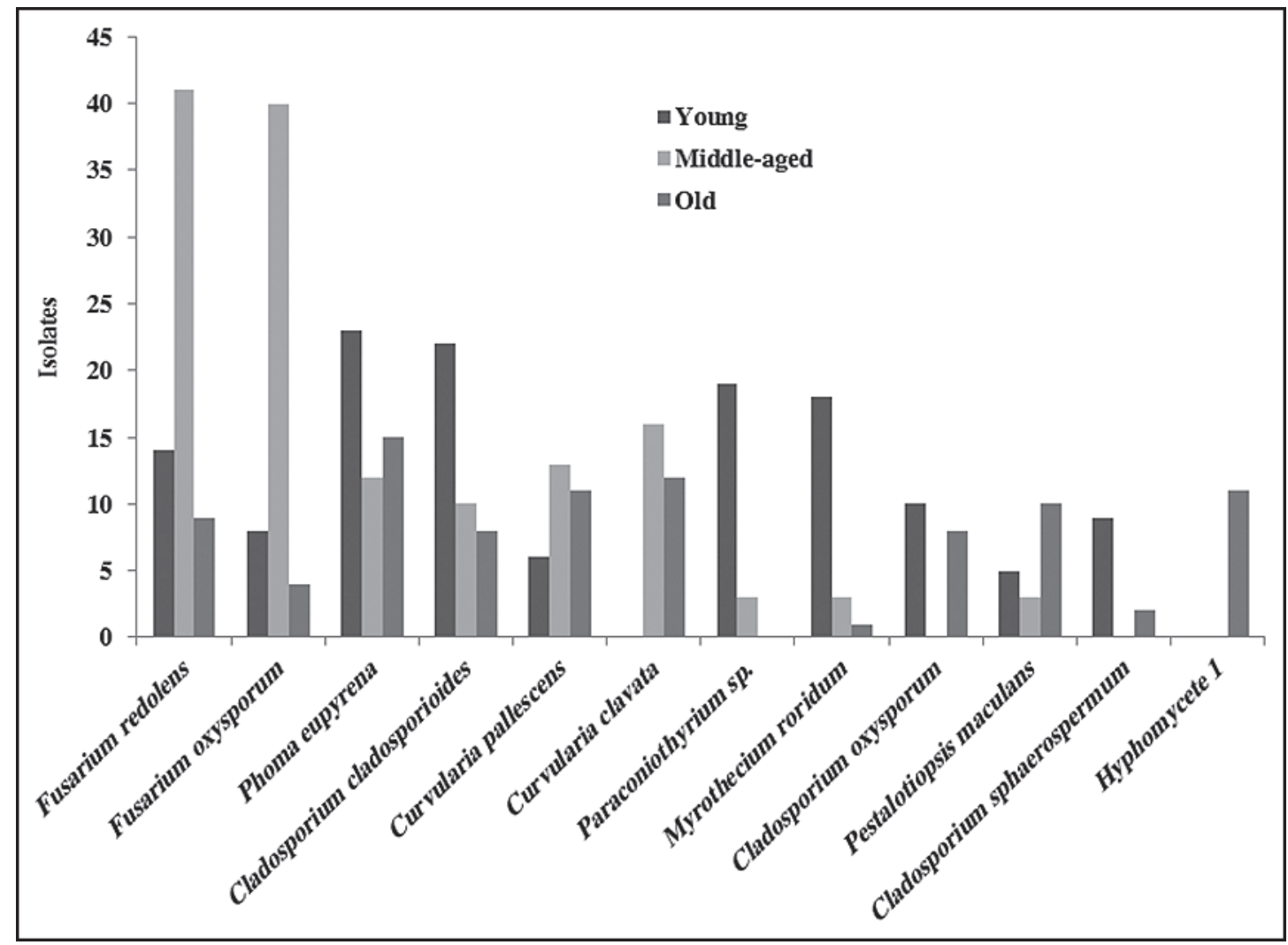

Figure 4: Fungal species with the highest number of isolates of the three biofilms a different stages of development on limestone.

middle-aged biofilm. Cladosporium oxysporum Berk. \& M.A. Curtis, Cladosporium sphaerospermum Penz. and Nodulisporium puniceum (Cooke \& Ellis) Deighton were found at a higher frequency in the young biofilm in comparison to the old biofilm. Curvularia verruculosa Tandon \& Bilgrami ex. M.B. Ellis was also more frequent in the middle-aged biofilm than the old biofilm. Meanwhile, the species Nodulisporium sylviforme Deighton and Paraphoma fimeti (Brunaud) Gruyter, Aveskamp \& Verkley were more dominant in the old biofilm than in the young biofilm. Finally, Myrothecium roridum Tode was more frequent in the old biofilm than in the middle-aged.

\section{DISCUSSION}

The fungal communities of biofilms occurring on limestone walls, considering time elapsed since wall construction, presented different degrees of microbial colonization. In the 5- and 10-year-old samples, colonization was evident by the green and black biomass of the biofilms that were visibly observed (Fig. 1), which is consistent with the findings of Adamson et al. (2013). The phototrophs colonizing such substrates are mainly composed of filamentous cyanobacteria and cocoidal bacteria (Scheerer et al., 2009); these have been found on Mayan buildings in the Yucatan peninsula (Ortega-Morales et al., 1999; 2013). The metabolic products of these organisms provide nutritional support for the establishment of heterotrophic communities, including fungi, allowing for their colonization (De la Torre et al., 1991).

The fungal composition was dominated by species of the Ascomycota class, which are common in rock substrates; these Hyphomicetous asexual species are able to colonize rocks during the first year of exposure (Ruibal et al., 2008; Gleeson et al., 2010; Hallman et al., 2011; Gómez-Cornelio et al., 2012). In contrast, Coelomycetous species are known to colonize limestone in Mediter- 
ranean regions (Wollenzien et al., 1995). The identification of only one taxon belonging to Basidiomycota may indicate that this group of organisms is not common in biofilms developing on limestone, as confirmed by Tang and Lian (2012), who used culture-independent methods; however, species of this group may exist in sterile form. The genera Aspergillus, Cladosporium, Curvularia, Fusarium, Microsphaeropsis, Myrothecium, Nodulisporium, Paraconiothyrium and Phoma are common in subtropical environments and in this study (Table 2). For other regions, some of the most common, dominant genera that have been reported on epilithic substrates include Alternaria, Cladosporium, Fusarium, Penicillium, Phoma and Trichoderma (Wollenzien et al., 1995; Kumar and Kumar, 1999; Gorbushina and Krumbein, 2000; Urzì et al., 2001; Gorbushina et al., 2002). All these genera were also identified in this study, but only Cladosporium, Fusarium and Phoma were common and dominant. These differences may be dictated by environmental factors and the bioreceptivity of rocks.

Some isolates showed no reproductive structures $(8 \%)$ and were identified as Mycelia sterilia (25\% of morphospecies). However, few isolates belonged to these morphospecies, indicating that they are rare in the community (Table 2). The finding of sterile mycelia commonly occurs in microbiological studies of other substrates (Arias-Mota and Heredia-Abarca, 2014; Rocha et al., 2014; Khirilla et al., 2015), including limestone substrates (Gómez-Cornelio et al., 2012). These fungi may be classified according to morphotype based on their morphological characteristics (Paulus et al., 2003). The production of less complex structures and poorly elaborated reproduction systems may represent an adaptation strategy in order to conserve energy on certain substrates (Ruibal et al., 2005) due to lack of nutrients or water.

In this study, greater species richness was found in the old biofilm. However, in another study on sandstone, the highest number of species was reported for fresh biofilms in comparison to older biofilms (Lan et al., 2010). Meanwhile, in this study the Shannon evenness index showed that the species identified from isolates were al- most equitable in the old biofilm. The lowest evenness was obtained in the middle-aged biofilm, probably due to the presence of several dominant species, such as Curvularia lunata and Fusarium redolens (Table 3). We found higher diversity values in comparison to other studies on fungal communities colonizing distinct substrates of plant litter and endophytes (Collado et al., 2007; Reverchon et al., 2010). This diversity may be due to the establishment and accumulation of propagules on bare rock at a constant and rapid speed. For example, an increase of $9 \times 10^{2}$ to $7.5 \times 10^{5}$ colony-forming units was documented in only 11 weeks (Gorbushina and Krumbein, 2000). Under ideal environmental conditions, a high number of fungi could colonize and grow on limestone.

These results, in addition to those of a previous study that investigated the fungal communities on bare limestone (Gómez-Cornelio et al., 2012), suggest that the species richness of limestone in subtropical environments is high in comparison to other rock surfaces, that have been studied in Europe, for example, and in particular in the Mediterranean (De la Torre et al., 1991; Sterflinger and Prillinger, 2001; Gorbushina et al., 2002; Ruibal et al., 2005; Hallman et al., 2011). Although the intrinsic characteristics of rock, such as its mineral composition, porosity and roughness, have been reported to influence the colonization of microbial communities (Guillitte, 1995; Burford et al., 2003; Lan et al., 2010), the study of Tomaselli et al. (2000) did not find a relationship among existing organisms and the petrographic characteristics of rock. In this study, the high values of species richness and diversity may be attributed to favorable environmental factors in the subtropics (Table 1) and time elapsed since initial colonization (Gaylarde and Gaylarde, 2005; Mihajlovski et al., 2014).

Furthermore, the Jaccard's similarity index showed low values of similarity among fungal communities corresponding to different ages. This is notable in considering that the middle-aged and old biofilms were located less than $1 \mathrm{~km}$ from each other. These biofilms also had a similar chromatic aspect; therefore, one might expect a high degree of similarity. Meanwhile, the young biofilm 
was located at an approximate distance of 10.5 kilometers from the other biofilms and had a visible although incipient colonization of microorganisms. Microclimatic differences present at each site may contribute towards the formation of a unique and particular mycobiota (Mihajlovski et al., 2014). Our results differed from those reported for sandstone, in which no differences in eukaryotic composition were found between fresh and young biofilms (Lan et al., 2010). However, to the contrary, on a serpentine substrate fungal communities were found to have low similarity (Daghino et al., 2012). These findings highlight that the community composition and diversity of fungi are not always determined by substrate bioreceptivity.

Based on the fungi that emerged from the analyzed particles that were scraped from biofilms on limestone, we were able to evaluate the fungal network and find a high proportion of active mycelium. The use of an oligotrophic culture medium (CCOA) allowed for the characterization of the cultivable fungi, which represented a complete, diverse and functional community (Ruibal et al., 2005). Although many of these species require water to grow, such as species of the Stachybotrys genus (Jain et al., 2009), the products of the extracellular matrix and the retention of water in rock pores could allow for the growth of water-demanding species. A large variety of specialized fungi were observed in this study; these species could represent an autochthonous community specific to limestone, a substrate with limited nutrients. However, due to the intrinsic environmental conditions of the tropics, nutrients may be transported by air and deposited on the rock as dust (Kumar and Kumar, 1999; Gorbushina and Krumbein, 2000; Ruibal et al., 2009), thereby providing conditions for the colonization of specialized fungal species in the production of exopolymers allowing fungi to adhere to the surfaces; some fungi are also capable of biomineralizing limestone. Furthermore, many fungi are able to successfully establish on limestone since they precipitate calcium; these represent the main sink of toxic forms of calcium in the soil or other environments (Sterflinger, 2000). Hence, fungi are essential members of the microbial communities that develop in the biofilms of limestone.

The composition of fungal communities may also be influenced by surrounding substrates (Urzì et al., 2001; Hallman et al., 2011). As previously mentioned, we identified the substrates previously reported in the literature for the 124 fungi that were identified in our study at the species level. A high proportion of fungi may have originated from soil or plants, and to a lesser extent, from decomposing litter and/or wood. Also, these fungal species could come from other rocks or from spores suspended in air or water (Fig. 2). However, the establishment and development of fungi over a period of time may be determined by the interactions of species with their environmental conditions, such as relative humidity and temperature (Gorbushina and Krumbein, 2000; Gorbushina et al., 2005), in addition to the bioreceptivity of the substrate. The variety of fungi reported for other surrounding substrates confirms that a large quantity of propagules may potentially reach and colonize rock surfaces. Therefore, according to our findings, limestone surfaces could act as a reservoir of fungal species and function as a fungal source via the dispersion of species under ideal conditions.

Most species isolated from biofilms occurred only once or twice, and few species showed dominance. The isolation technique used in this study may promote the growth of rare and uncommon species; these results do concur with those found by other authors who used both taxonomic and molecular identification techniques (Collado et al., 2007; Ruibal et al., 2008; Gómez-Cornelio et al., 2012). Additionally, the abundance of dominant species of the three analyzed biofilms was variable and may be determined by time of exposure, similar to the variations observed in Lan et al. (2010) in the microorganism community of old and fresh biofilms.

The fungal community contained melanin at some or all of their reproductive stages (74\% of the species); this concurs with the reports of other authors, in which the dominant fungi isolated from monuments also contained pigmentation (Sterflinger and Krumbein, 1997; Gorbushina et al., 2002; Lan et al., 2010). Pigmentation in fungi may have different functions or result from the 
environmental conditions experienced on the rock surface (Scheerer et al., 2009; Hallman et al., 2011). Therefore, melanized communities of fungi have been shown to occur at a high frequency on rock surfaces, although composition may differ from one community to another, as mentioned in the results.

Moreover, fungal propagules are capable of quickly colonizing rock surfaces. Gorbushina and Krumbein (2000) have suggested that fluctuations in environmental conditions, such as those that lead to deficits in nutrients and water on the rock surfaces, promote changes in the diversity of the fungal community. In our results the environmental conditions and the characteristics of the limestone substrate of the biofilms were similar; thus one would expect to find a relationship among the studied fungal communities. However, it may be necessary to study the roles of dominant species during succession of fungal communities. For example, it has been shown that the hyphae of common fungal species biomineralize the surface of the limestone (Burford et al., 2006) and thus lead to subsequent changes. In this study the composition of the fungal community is likely determined by time of exposure to the environment and species interactions, which may then facilitate or inhibit colonization by other species, leading to changes in the composition of the fungal chronosequence associated with limestone (Fryar, 2002). During this process, the functional properties of fungi on the limestone may also be potentially affected.

\section{CONCLUSIONS}

In this study, the fungal communities immersed in biofilms were different at each stage of development, defined by the time of exposure of the limestone substrate to the environment. Although the mineral composition of rock substrates has been found to have a certain degree of influence on the structure of fungal communities, in this study limestone samples with similar characteristics of bioreceptivity, such as rock color, roughness and porosity, were selected. Environmental conditions were also similar across sites in the city of Campeche, Mexico. Therefore, in addition to time of exposure, the differentiation in the community structu- re and diversity of fungi in this study may be determined by the interactions among the species of each biofilm; this should be confirmed by subsequent studies. A particular species composition was isolated in each biofilm corresponding to a different developmental stage, although a common pool of hyaline and melanized fungi appear to colonize rock with great success and may have specific functions on the rock substrate. In future studies, biotic factors, including interactions among bacterial, fungal and algal species, should be studied in order to determine their influence on the structure of the fungal community.

\section{ACKNOWLEDGEMENTS}

We are grateful to Julio C. Rojas León and Hugo Perales Rivera for their comments on an early version of the manuscript. This research was supported by institutional funding from El Colegio de la Frontera Sur and the Universidad Autónoma de Campeche. We extend our thanks to the Comisión Nacional del Agua for the provision of meteorological data and to the Consejo Nacional de Ciencia y Tecnología for the doctoral scholarship awarded to S.G.C. We thank two anonymous reviewers and the editor of the manuscript for suggested improvements; also thanks to Allison Marie Jermain for reviewing the English version of the manuscript.

\section{LITERATURE CITED}

Adamson, C., S., McCabe, P. A. Warke, D. McAllister and B. J. Smith. 2013. The influence of aspect on the biological colonization of stone in Northern Ireland. International Biodeterioration \& Biodegradation 84: 357-366. DOI: http://dx.doi.org/10.1016/j.ibiod.2012.05.023

Arias-Mota, R. M. and G. Heredia-Abarca. 2014. Fungal diversity in coffee plantation systems and in a tropical montane cloud forest in Veracruz, Mexico. Agroforestry System 88: 921-933. DOI: http://dx.doi.org/10.1007/s10457-0149736-z

Barberousse, H., R. J. Lombardo, G. Tell and A. Couté. 2006. Factors involved in the colonisation of building facades by algae and cyanobacteria in France. Biofouling 22: 6977. DOI: http://dx.doi.org/10.1080/08927010600564712 
Bills, G. F., M. Christensen, M. Powell and R. G. Thorn. 2004. Saprobic soil fungi. In: Mueller, G. M., G. F. Bills and M. S. Foster (ed.). Biodiversity of fungi: inventory and monitoring methods. Elsevier. Amsterdam, The Netherlands. pp. 271-301.

Boerema, G. H., J. Gruyter, M. E. Noordeloos and M. E. C. Hamers. 2004. Phoma identification manual. Differentiation of specific and infraspecific taxa in culture. CABI publishing. London, UK. 470 pp.

Booth, C. 1971. The genus Fusarium. Commonwealth Mycological Institute. Kew, UK. 237 pp.

Burford, E. P., M. Kierans and G. M. Gadd. 2003. Geomycology: fungal growth in mineral substrata. Mycologist 17: 98-107. DOI: http://dx.doi.org/10.1017/ S0269915X03003112

Burford, E. P., S. Hillier and G. M. Gadd. 2006. Biomineralization of fungal hyphae with calcite $\left(\mathrm{CaCO}_{3}\right)$ and calcium oxalate mono-and dihydrate in carboniferous limestone microcosms. Geomicrobiology Journal 23: 599-611. DOI: http://dx.doi.org/10.1080/01490450600964375

Chertov, O. G., A. A. Gorbushina and B. Deventer. 2004. A model for microcolonial fungi growth on rock surfaces. Ecological Modelling 177: 415-426. DOI: http://dx.doi. org/10.1016/j.ecolmodel.2004.02.011

Collado, J., G. Platas, B. Paulus and G. Bills. 2007. High-throughput culturing of fungi from plant litter by a dilution to-extinction technique. FEMS Microbiology Ecology 60: 521-533. DOI: http://dx.doi.org/10.1111/ j.1574-6941.2007.00294.x

Colwell, R. K. 2013. EstimateS: statistical estimation of species richness and shared species from samples. Version 9 - User's guide and application.

Daghino, S., C. Murat, E. Sizzano, M. Girlanda and S. Perotto. 2012. Fungal diversity is not determined by mineral and chemical differences in serpentine substrates. PLoS One 7: e44233. DOI: http://dx.doi.org/0.1371/journal. pone. 0044233

De la Torre, M. A., G. Gómez-Alarcón, P. Melgarejo and C. Saiz-Jimenez. 1991. Fungi in weathered sandstone from Salamanca cathedral, Spain. Science of the Total Environment 107: 159-168. DOI: http://dx.doi.org/10.1016/00489697(91)90257-F
Domsch, K. H., W. Gams and T. H. Anderson. 2007. Compendium of soil fungi. 2 nd ed. Academic press. London, UK. $672 \mathrm{pp}$.

Ellis, M. B. 1971. Dematiaceous hyphomycetes. CAB. Kew, UK. 608 pp.

Ellis, M. B. 1976. More dematiaceous hyphomycetes. CAB. Kew, UK. 507 pp.

Fryar, S. C. 2002. Fungal succession or sequence of fruit bodies? Fungal Diversity 10: 5-10.

Gadd, G. M. 2007. Geomycology: biogeochemical transformations of rocks, minerals, metals and radionuclides by fungi, bioweathering and bioremediation. Mycological Research 111: 3-49. DOI: http://dx.doi.org/10.1016/j.mycres.2006.12.001

Gaylarde, C. C. and P. M. Gaylarde. 2005. A comparative study of the major microbial biomass of biofilms on exteriors of buildings in Europe and Latin America. International Biodeterioration \& Biodegradation 55: 131-139. DOI: http:// dx.doi.org/10.1016/j.ibiod.2004.10.001

Gleeson, D. B., K. Melville, F. McDermott, N. Clipson and G. M. Gadd. 2010. Molecular characterization of fungal communities in sandstone. Geomicrobiology Journal 27: 559571. DOI: http://dx.doi.org/10.1080/01490451003702982

Gómez-Cornelio, S., J. Mendoza-Vega, C. C. Gaylarde, M. Reyes-Estebanez, A. Morón-Ríos, S. C. De la Rosa-García and B. O. Ortega-Morales. 2012. Succession of fungi colonizing porous and compact limestone exposed to subtropical environments. Fungal Biology 116: 1064-1072. DOI: http://dx.doi.org/10.1016/j.funbio.2012.07.010

Gorbushina, A. A. and W. E. Krumbein. 2000. Rock dwelling fungal communities: diversity of life styles and colony structure. In: J. Seckbach (ed.). Journey to diverse microbial worlds. Kluwer, Amsterdam. pp. 317-334.

Gorbushina, A. A., N. N. Lyalikova, D. Y. Vlasov and T. V. Khizhnyak. 2002. Microbial communities on the monuments of Moscow and St. Petersburg: Biodiversity and trophic relations. Microbiology 71: 350-356. DOI: http://dx.doi. org/10.1023/A:1015823232025

Gorbushina, A. A., A. Beck and A. Schulte. 2005. Microcolonial rock inhabiting fungi and lichen photobionts: evidence for mutualistic interactions. Mycological Re- 
search 109: 1288-1296. DOI: http://dx.doi.org/10.1017/ S0953756205003631

Gorbushina, A. A. 2007. Life of the rock. Environmental Microbiology 9: 1613-1631. DOI: http://dx.doi.org/10.1111/ j.1462-2920.2007.01301.x

Guillitte, O. 1995. Bioreceptivity: a new concept for building ecology studies. Science of the Total Environment 167: 215-220. DOI: http://dx.doi.org/10.1016/00489697(95)04582-L

Hallman, C., D. Fritzlar, L. Stannek and M. Hoppert. 2011. Ascomycete fungi on dimension Stone of the "Burg Gleichen”, Thuringia. Environmental Earth Sciences 63: 1713-1722. DOI: http://dx.doi.org/10.1007/s12665-0111076-y

Jain, A., S. Bhadauria, V. Kumar and R. S. Chauhan. 2009. Biodeterioration of sandstone under the influence of different humidity levels in laboratory conditions. Building and Environment 44: 1276-1284. DOI: http://dx.doi. org/10.1016/j.buildenv.2008.09.019

Khiralla, A., I. Mohamed, J. Thomas, B. Mignard, R. Spina, S. Yagi and D. Laurain-Mattar. 2015. A pilot study of antioxidant potential of endophytic fungi from some Sudanese medicinal plants. Asian Pacific Journal of Tropical Medicine 8: 701-704. DOI: http://dx.doi.org/10.1016/j. apjtm.2015.07.032

Klich, M. A. 2002. Identification of common Aspergillus species. The Centraalbureau Voor Schimmelcultures. Utrecht, The Netherlands. 122 pp.

Kumar, R. and A. V. Kumar. 1999. Biodeterioration of stone in tropical environments: An overview. The Getty Conservation Institute. Los Angeles, USA. 85 pp.

Lan, W., H. Li, W. D. Wang, Y. Katayama and J. D. Gu. 2010. Microbial community analysis of fresh and old microbial biofilms on Bayon temple sandstone of Angkor Thom, Cambodia. Microbial Ecology 60: 105-115. DOI: http:// dx.doi.org/10.1007/s00248-010-9707-5

Mihajlovski, A., D. Seyer, H. Benamara, F. Bousta and P. Di Martino. 2014. An overview of techniques for the characterization and quantification of microbial colonization on stone monuments. Annals of Microbiology 65: 12431255. DOI: http://dx.doi.org/10.1007/s13213-014-0956-2
Miller, A. Z., P. Sanmartín, L. Pereira-Pardo, A. Dionísio, C. Saiz-Jimenez, M. F. Macedo and B. Prieto. 2012. Bioreceptivity of building stones: A review. Science of the Total Environment 426: 1-12. DOI: http://dx.doi.org/10.1016/j. scitotenv.2012.03.026

Ortega-Morales, O., G. Hernandez-Duque,L. Borges-Gomezand J. Guezennec. 1999. Characterization of epilithic microbial communities associated with Mayan stone monuments in Yucatán, México. Geomicrobiology Journal 16: 221232. DOI: http://dx.doi.org/10.1080/014904599270604

Ortega-Morales, B. O., C. C. Gaylarde, A. Anaya-Hernández, M. J. Chan-Bacab, S. C. De la Rosa-García, D. Arano-Recio and J. L. Montero-Muñoz. 2013. Orientation affects Trentepohlia-dominated biofilms on Mayan monuments of the Rio Bec style. International Biodeterioration \& Biodegradation 84: 351-356. DOI: http://dx.doi. org/10.1016/j.ibiod.2012.07.014

Paulus, B. and P. Gadek. 2003. Estimation of microfungal diversity in tropical rainforest leaf litter using particle filtration: the effects of leaf storage and surface treatment. Mycological Research 107: 748-756. DOI: http://dx.doi. org/10.1017/S0953756203007913

Pitt, J. I. 2000. A laboratory guide to common Penicillium species, 3rd ed. Food Science Australia. Sydney, Australia. $197 \mathrm{pp}$.

Reverchon, F., P. M. Ortega-Larrocea, J. Pérez-Moreno, V. M. Peña-Ramírez and C. Siebe. 2010. Changes in community structure of ectomycorrhizal fungi associated with Pinus montezumae across a volcanic soil chronosequence at Sierra Chichinautzin, Mexico. Canadian Journal of Forest Research 40: 1165-1174. DOI: http://dx.doi.org/10.1139/X10-062

Rocha, S. L., V. L. Jorge, T. M. Della Lucia, R. W. Barreto, H. C. Evans and S. L. Elliot. 2014. Quality control by leaf-cutting ants: evidence from communities of endophytic fungi in foraged and rejected vegetation. Arthropod-Plant Interactions 8: 485-493. DOI: http://dx.doi. org/10.1007/s11829-014-9329-9

Ruibal, C., G. Platas and G. F. Bills. 2005. Isolation and characterization of melanized fungi from limestone formations in Mallorca. Mycological Progress 4: 23-38. DOI: http:// dx.doi.org/10.1007/s11557-006-0107-7 
Ruibal, C., G. Platas and G. F. Bills. 2008. High diversity and morphological convergence among melanised fungi from rock formations in the Central Mountain System of Spain. Persoonia 21: 93-110. DOI: http://dx.doi. org $/ 10.3767 / 003158508 X 371379$

Ruibal, C., C. Gueidan, L. Selbmann, et al. 2009. Phylogeny of rock-inhabiting fungi related to Dothideomycetes. Studies in Mycology 64: 123-133. DOI: http://dx.doi. org/10.3114/sim.2009.64.06

Scheerer, S., O. Ortega-Morales and C. Gaylarde. 2009. Microbial deterioration of stone monuments - an updated overview. Advances in Applied Microbiology 66: 97-139. DOI: http://dx.doi.org/10.1016/S0065-2164(08)00805-8

Seifert, K., G. Morgan-Jones, W. Gams and B. Kendrick. 2011. The genera of Hyphomycetes. CBS-KNAW Fungal Biodiversity Center. London, UK. 997 pp.

Sterflinger, K. and W. E. Krumbein. 1997. Dematiaceous fungi as a major agent for biopitting on Mediterranean marbles and limestones. Geomicrobiology Journal 14: 219-231. DOI: http://dx.doi.org/10.1080/01490459709378045

Sterflinger, K. 2000. Fungi as geologic agents. Geomicrobiology Journal 17: 97-124. DOI: http://dx.doi. org $/ 10.1080 / 01490450050023791$

Sterflinger, K. and H. Prillinger. 2001. Molecular taxonomy and biodiversity of rock fungal communities in an urban environment (Vienna, Austria). Antonie van Leeuwenhoek 80: 275-286. DOI: http://dx.doi. org/10.1023/A:1013060308809
Sterflinger, K., D. Tesei and K. Zakharova. 2012. Fungi in hot and cold deserts with particular reference to microcolonial fungi. Fungal Ecology 5: 453-462. DOI: http://dx. doi.org/10.1016/j.funeco.2011.12.007

Sutton, B. C. 1980. The Coelomycetes. CABI Published. London, UK. 696 pp.

Tang, Y. and B. Lian. 2012. Diversity on endolithic fungal communities in dolomite and limestone rocks from Nanjiand Canyon in Guizhou karst area, China. Canadian Journal of Microbiology 58: 685-693. DOI: http://dx.doi. org/10.1139/w2012-042

Tomaselli, L., G. Lamenti, M. Bosco and P. Tiano. 2000. Biodiversity of photosynthetic microorganisms dwelling on stone monuments. International Biodeterioration \& Biodegradation 46: 251-258. DOI: http://dx.doi.org/10.1016/ S0964-8305(00)00078-0

Urzì, C., F. De Leo, P. Salamone and G. Criseo. 2001. Airborne fungal spores colonising marbles exposed in the terrace of Mesina Museum, Sicily. Aerobiologia 17: 11-17. DOI: http://dx.doi.org/10.1023/A:1007652300354

Wollenzien, U., G. S. De Hoog, W. E. Krumbein and C. Urzì. 1995. On the isolation of microcolonial fungi occurring on and in marble and other calcareous rocks. Sciences of the Total Environment 167: 287-294. DOI: http://dx.doi. org/10.1016/0048-9697(95)04589-S 